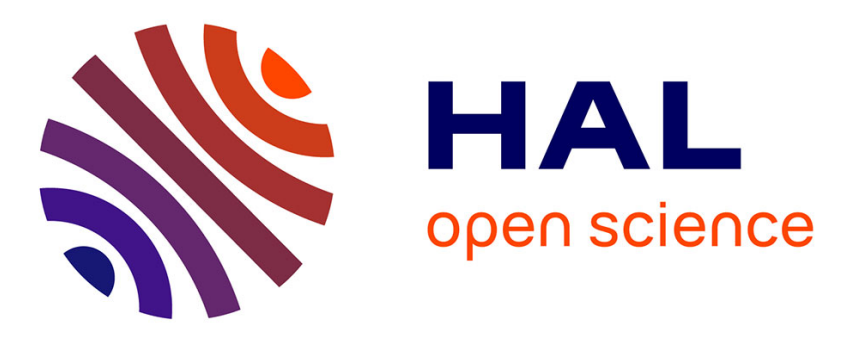

\title{
Concomitant carriage of KPC-producing and non-KPC-producing Klebsiella pneumoniae ST512 within a single patient
}

Agnès Jousset, Rémy Bonnin, Julie Takissian, Delphine Girlich, Liliana

Mihaila, Nicolas Cabanel, Laurent Dortet, Philippe Glaser, Thierry Naas

\section{To cite this version:}

Agnès Jousset, Rémy Bonnin, Julie Takissian, Delphine Girlich, Liliana Mihaila, et al.. Concomitant carriage of KPC-producing and non-KPC-producing Klebsiella pneumoniae ST512 within a single patient. Journal of Antimicrobial Chemotherapy, 2020, 75 (8), pp.2087-2092. 10.1093/jac/dkaa137 . hal-03102257

\section{HAL Id: hal-03102257 \\ https://hal.science/hal-03102257}

Submitted on 31 Mar 2021

HAL is a multi-disciplinary open access archive for the deposit and dissemination of scientific research documents, whether they are published or not. The documents may come from teaching and research institutions in France or abroad, or from public or private research centers.
L'archive ouverte pluridisciplinaire HAL, est destinée au dépôt et à la diffusion de documents scientifiques de niveau recherche, publiés ou non, émanant des établissements d'enseignement et de recherche français ou étrangers, des laboratoires publics ou privés. 


\section{Concomitant carriage of KPC-producing and non-KPC-producing Klebsiella pneumoniae ST512 within a single patient}

Agnès B. JOUSSET, ${ }^{1,2,3,4}$ Rémy A. BONNIN, ${ }^{1,3,4}$ Julie TAKISSIAN, ${ }^{1}$ Delphine GIRLICH, ${ }^{1,4}$ Liliana MIHAILA, ${ }^{2}$ Nicolas CABANEL, ${ }^{4}$ Laurent DORTET, $, 1,3,4$ Philippe GLASER, ${ }^{4,5}$ Thierry NAAS. ${ }^{1,2,3,4^{*}}$

1EA7361 "Structure, dynamic, function and expression of broad spectrum $\beta$ lactamases", Université Paris Sud, Université Paris Saclay, LabEx Lermit, Faculty of Medicine, Le Kremlin-Bicêtre, France

${ }^{2}$ Bacteriology-Hygiene unit, Assistance Publique/Hôpitaux de Paris, Bicêtre Hospital, Le Kremlin-Bicêtre, France

${ }^{3}$ Associated French National Reference Center for Antibiotic Resistance: Carbapenemase-producing Enterobacteriaceae, Le Kremlin-Bicêtre, France ${ }^{4}$ Evolution and Ecology of Resistance to Antibiotics Unit, Institut Pasteur - APHP Université Paris-Sud, Paris, France

${ }^{5}$ CNRS UMR3525, Paris, France

Short title: Within-host diversity of K. pneumoniae ST512

Main text: 1923 words

Synopsis: 249 words

Table: 1

Figure: 1

${ }^{*}$ Corresponding author mailing address: Service de Bactériologie-Hygiène, Hôpital de Bicêtre, 78 rue du Général Leclerc, 94275 Le Kremlin-Bicêtre Cedex, France.

Fax: + 33145216340

E-mail: thierry.naas@aphp.fr 


\section{Synopsis}

Background: KPC-producing Klebsiella pneumoniae $(K p)$ of the clonal group 258 are prominent in the health care settings in many regions of the world. The blakPC gene is mostly carried by a multi-replicon IncFIlk-IncFI plasmid suspected to be highly compatible and stable in this genetic background. Here, we analysed the genetic diversity of a ST512 Kp population in a single patient.

Material and methods: Twelve $K p$ isolates $(n=5$ from urine samples and $n=7$ from rectal swabs) were recovered from one patient over a two-months period. Antimicrobial susceptibility testing, plasmid extraction and WGS were performed on all isolates. The first $K p$ isolate D1 was used as reference for phylogenetic analysis.

Results: Antimicrobial susceptibility testing, plasmid analysis and WGS revealed concomitant carriage of carbapenem-resistant and carbapenem-susceptible $\mathrm{Kp}$ isolates of the sequence type 512 , with the absence of the entire blakPC-carrying plasmid in the susceptible population. Furthermore, 14 other genetic events occurred within the genome including three chromosomal deletions (of $71 \mathrm{~kb}, 33 \mathrm{~kb}$ or $11 \mathrm{nt}$ insize), two different insertions of SKpn26 and 9 SNPs. Interestingly, most of the events occurred in the same chromosomal region that has been deleted independently several times probably after homologous recombination involving 259 nt in-size repeated sequences.

Conclusions: Our study revealed the first case of in-vivo blakPC-carrying plasmid curing and a wide in-patient genetic diversity of a single Kp ST512 clone over a short period of carriage. This within-patient diversity must be taken into account when characterizing transmission chains using WGS during nosocomial outbreaks. 


\section{Introduction}

Carbapenem-resistant Klebsiella pneumoniae has emerged as a formidable threat in health care facilities. ${ }^{1} \underline{K}$ lebsiella pneumoniae carbapenemase (KPC) belongs to Ambler's Class A and has emerged globally in Enterobacterales in the early 2000's. ${ }^{2}$ The worldwide spread of KPC is multifactorial and has been related to the diffusion of a particular clonal group (CG), the CG258 defined by few single locus variants (SLV), ST258, ST11, and ST512, being the most predominant ones. ${ }^{3}$ Notably, the emergence of blakPC-3 gene in Italy has been related to the spread of the ST512 isolates. ${ }^{4,5}$ Other genetic features associated to its spread are related to the genetic structure, a class II transposon containing the blakPC gene named Tn4401-like, and a conjugative plasmid of IncFIIK type..$^{2,6,7}$

After the worldwide dissemination of these successful clones and clonalcomplex since 2000, all reports of $K$. pneumoniae (Kp) of the CC258 refer to KPCproducing strains with the exception of a study that retrospectively analysed carbapenem-susceptible Kp collection strains isolated between 1999 and 2013 in New York City and some of them belonged to the ST258. ${ }^{8}$

Whole-Genome Sequencing has emerged as a powerful tool to study bacterial evolution. Evolution of the genome of KPC-Kp-ST258 during long-term human colonization revealed complex plasmid rearrangements and genome plasticity. ${ }^{9,10}$ However, little is known about the genetic diversity that resides in the gastro-intestinal tract within a single bacterial population at a specific time point.

Here, we described the concomitant carriage of blakPc-positive carbapenemresistant and blakPC-negative carbapenem-susceptible Kp-ST512 clinical isolates in a single patient. Our objectives were to analyse the genetic diversity of a KPC-Kp isolate and to appreciate the cohabitation of several sub-populations of Kp-ST512. 
Material and methods

Bacterial isolates, MICs and growth conditions. Twelve clinical isolates of $K$. pneumoniae were isolated from a single patient over a two-months period. The isolates were obtained from rectal swabs $(n=7)$ or urine samples $(n=5)$ and were named after the sampling date, D1 being the first isolate and D54 the one being isolated 54 days later. From rectal swabs, K. pneumoniae were obtained after growth on selective media supplemented with carbapenems (ChromID ${ }^{\circledR}$ CARBA SMART, bioMérieux, Marcy L'Etoile, France) whereas urine samples were spread on non- selective chromogenic media (UriSelect ${ }^{\mathrm{TM}}$ Bio-Rad, Marnes-La-Coquette, France).

Antimicrobial susceptibilities were determined by the disc diffusion method on MuellerHinton $(\mathrm{MH})$ agar (Bio-Rad), and were interpreted according to EUCAST guidelines. ${ }^{11}$ MICs for carbapenems, ceftazidime, tigecycline, ciprofloxacin and aminoglycosides were determined by Etest (bioMérieux), and for colistin by broth microdilution (Sensititre ${ }^{\mathrm{TM}}$ Thermofisher, France).

Genomic analysis. Total DNA was extracted from colonies using the Ultraclean ${ }^{12}$ Microbial DNA Isolation Kit (MO BIO Laboratories, Ozyme, Saint-Quentin, France) following the manufacturer's instructions. The DNA library was prepared using the Nextera XT-v2 kit (Illumina, Paris, France) and then run on HiSeq Illumina system (2x150-bp paired-end). Full sequence of Kp D1 genome was obtained using PacBio and Illumina's sequencing technologies. Assembly was performed using the RS_HGAP_Assembly.3 protocol from the SMRT analysis toolkit v2.3 and with Canu. ${ }^{12}$ The consensus sequence was polished with Quiver, and manually corrected by 107 mapping Illumina reads using Breseq. ${ }^{12}$ The acquired antimicrobial resistance genes 
109 (https://cge.cbs.dtu.dk/services/ResFinder/). ${ }^{13}$ The genome was annotated using PROKKA. ${ }^{14}$

SNP analysis was performed by mapping the reads from each genome (D2 to

112 D54) against Kp D1 genome used as reference. Variants, SNP, insertion and deletion

113 were detected by using BRESEQ ${ }^{12}$ or the variant detection tool of CLC genomic workbench v12.0 (Qiagen, Les Ulis, France).

116 Plasmid content analysis. Plasmids were extracted using the Kieser's extraction

117 method and subsequently analysed by electrophoresis on a $0.7 \%$ agarose gel. ${ }^{15}$

Nucleotide sequence accession number. The whole genome sequences generated in the study have been submitted to the Genbank nucleotide sequence database under accession numbers detailed in Table 1.

Ethics. This study was conducted in accordance with the Declaration of Helsinki and national standards. Signed statement of informed consent was obtained from the 125 patient.

\section{Results}

Case report. In 2015, a patient suffered from acute pancreatitis (Balthazar

130 score E) due to gallstones during a stay in Italy. The patient was hospitalized in Italy 131 for ten days, during which no nutrition was given to rest the pancreas and bowels.

132 Food was then reintroduced through a nasogastric tube, and the evolution was 
133 favorable with exclusive enteric nutrition. The patient was repatriated to France for

134 further medical care. Upon admission, screening for intestinal carriage of 135 carbapenemase-producing Enterobacterales (CPE) allowed to identify the presence 136 of a KPC-producing Kp (isolate D1), likely acquired during its hospitalization in Italy. A

137 cholecystectomy was performed 10 weeks after the acute episode and no complication

138 occurred. During the two months of follow-up in France, 12 K. pneumoniae isolates (8 139 isolates KPC+ and 4 KPC-) were recovered from screening or urine samples (Figure $1401 \mathrm{~A})$.

Susceptibility testing and resistome. Susceptibility testing of the twelve

143 isolates recovered revealed two different phenotypes regarding $\beta$-lactams but the same co-resistances. WGS revealed in all genomes three aminoglycosides modifying enzymes (aph(3')-la, aac(6')-lb, aadA2), the natural fosfomycin resistance gene (fosAlike), catA1-like, dfrA12 and sul1 conferring resistance to chloramphenicol,

147 trimethoprime and sulfamides respectively. A substitution in GyrA (S83I) was also 148 identified conferring resistance to fluoroquinolones. OmpK35 porin was inactivated by an insertion at position 121 of the gene $(+G)$, leading to an early STOP codon in the 150 protein. In OmpK36, two amino-acids are inserted at position 135 (+Asp) and 136 151 (+Gly) in comparison to wild-type sequence (NC_016845.1). This OmpK36 variant is 152 known to contribute to increase the MICs for carbapenems in Kp of CG258. ${ }^{16}$ Eight isolates were resistant to carbapenems (MICs for imipenem from 4 to 8 $\mathrm{mg} / \mathrm{L}$, MICs for meropenem and ertapenem $>32 \mathrm{mg} / \mathrm{L}$ ) whereas four isolates were susceptible to broad-spectrum cephalosporins and carbapenems (MICs for imipenem at $0.125 \mathrm{mg} / \mathrm{L})$. The content of $\beta$-lactamase-encoding genes differed between isolates.

157 WGS revealed that all carbapenem-resistant $K p$ contained three acquired $\beta$ - 
158 lactamases genes: blakPC-3 carried by a transposon Tn4401a, blaTEM-1, blaoxA-9 (not

159 functional due to premature stop codon) carried by a multireplicon IncFIB-IncFIlk

160 plasmid of $113,639 \mathrm{bp}$ (pKpQIL-like) and the naturally chromosome-encoded blasHV-11

161 gene. Carbapenem-susceptible isolates possessed only the blasHv gene.

162 MLST analysis indicated that all Kp (KPC+ and KPC-) belonged to the ST512,

163 suggesting that the two populations of Kp ST512 seem to differ only by the presence

164 or absence of the Tn4401a or of the whole blakpc-carrying plasmid. In order to

165 distinguish between these two hypotheses, plasmid extractions analysed by

166 electrophoresis revealed that a c.a. $100 \mathrm{~kb}$ plasmid was missing in all KPC- isolates

167 (Figure 1B).

Genomic analysis and phylogeny. To establish whether a gain or a loss of the blakPC-carrying plasmid occurred in the Kp ST512 population, SNPs and genetic events underlying in vivo evolution analyses were performed using isolate D1 as 172 reference (Figure $1 \mathrm{C})$.

Even though the 12 isolates were highly related, a total of 15 different genetic events were identified: the loss of the whole blakpc-carrying plasmid (in 4 isolates), three different deletions of chromosomal regions $(71,959$ bp in-size in 5 isolates, 33,752 bp in-size in 2 isolates, and 11 bp in-size in two isolates), two different insertions

177 of a copy of ISKpn26 (at position 2,309,021 in 5 isolates and at position 4,117,119 in 178 one ) and 9 different SNPs (involving 7 isolates). Interestingly, the $33 \mathrm{~kb}$ deleted region 179 (from 4,106,137- 4,139,889) was part of the larger $71 \mathrm{~kb}$ deleted region (from $1804,068,121$ to $4,139,091)$. No mobile element could be evidenced surrounding these 181 two deleted regions, but the presence of three copies of Repeated Sequences (RS) of 182259 nt in-size were present (Figure 1.D). These regions share over $96 \%$ of nucleotide 
183 identity (Supplementary Figure). Recombination events involving RS1 and RS3, and

184 RS2 and RS3 are likely responsible for the deletions of the $71 \mathrm{~kb}$ and the $33 \mathrm{~kb}$ in-size 185 fragments respectively.

186 Comparison to deposited genomes in public databases indicates that D33-3

187 isolate is the closest to other Kp ST512 genomes. In the chromosome of D1 (KPC+),

188 a deletion of $11 \mathrm{nt}$ in-size occurred, and this deletion is also present in D33-2 (KPC-)

189 in addition to the insertion of a copy of ISKpn26. Furthermore, the loss of pKPC seems

190 to have occurred in another branch, between D33-3 (KPC+) and D19-2 (KPC-) (Figure

191 1C). These observations make highly likely the loss of the blakPC-carrying plasmid by

192 the KPC+ population rather than an acquisition by the KPC- population.

193

194 Discussion

195 An unexpected genetic and phenotypic variability of Kp ST512 in a single patient

196 was observed as a result of several unrelated genetic events. Concomitant isolation

197 of carbapenem-resistant and -susceptible isolates recovered over a short period of

198 time (two months) was due to the presence or not of the blakPC-3 carrying plasmid

199 (pKPC). In our study, this diversity was clearly underestimated by the use of selective

200 media for rectal samples that allow only the growth of carbapenem-resistant bacteria.

201 Hence the carbapenem -susceptible population could be identified only from urine

202 samples. Despite this major bias, three isolates from different branches of the

203 distribution were recovered the same day (D33) as a proof that this genetic diversity is 204 present in the patient's microbiota.

205 Interestingly, over the 15 genetic events described, 5 involved the same chromosomal 206 region (from 4068121 - 4139091) in 10 isolates. This region included over 60 CDS 207 and among them, the ramA gene (Accession KC843634) is either entirely deleted (in 
$20871 \mathrm{~kb}$ and in $33 \mathrm{~kb}$-deleted genomes), inactivated by a non-sense mutation, or 209 putatively transcriptionnally affected by the insertion of a copy of ISKpn26 in the

210 intergenic region, or by a $11 \mathrm{nt}$ deletion in the ramR regulator (Figure 1D). ramA, an

211 araC-family transcriptional regulator, is part of the $\operatorname{ramR}$-romA-ramA operon and is

212 involved in the expression of AcrAB efflux pump leading to increased MICs for

213 tigecycline and fluoroquinolones. We could not observe any correlation between MICs

214 for these antibiotics and any of the genetic events. However, since this region has been

215 inactivated several times by independent mechanisms, it is tempting to speculate that

216 its inactivation confers to this $\mathrm{Kp}$ ST512 a competitive advantage in that clinical context.

217 The genetic analysis indicated that a loss of the blakPc-3 carrying plasmid likely

218 occurred in the Kp ST512 population. pKPC is very close to the successful pKpQIL 219 plasmid $(99,98 \%$ of nucleotide identity, $100 \%$ of query coverage), the first KPC220 producing plasmid that had been sequenced in 2006 and known to have spread in all

221 Europe through its tight association to the CG258. ${ }^{17,18}$ Plasmid stability assay could

222 not evidence an increased capacity of Kp D1 to loose pKPC plasmid in comparison to

223 other Kp isolates carrying pKpQIL-like plasmids (data not shown). Previous genomic 224 analyses of KPC-Kp during long-term carriage have reported large plasmid 225 rearrangements, deletions of the entire Tn4401, or plasmid transfer between 226 Enterobacterales, but loss of the entire blakpc-carrying plasmid by a member of the $227 \mathrm{Kp}$-CG258 has never been reported to date..$^{9,19}$ The pKpQIL-like plasmids are thought 228 to be highly compatible with $\mathrm{Kp}$-CG258 genetic background and to have contributed to 229 the worldwide dissemination of KPC carbapenemase. ${ }^{2,20}$ Information regarding the 230 antibiotic selection pressure that occurred during the patient's hospitalization were 231 available (Figure 1A). Given the genetic support of resistance genes linked to these 232 antimicrobial agents, cefixime seems to be the solely molecule capable to maintain a 
233 selective pressure to prevent the loss of the KPC plasmid. But it has been prescribed

234 at Day 44, far after the isolation of the first non KPC-producer at Day 19. So most of

235 the patient's bacterial follow-up was done when no antimicrobial selective pressure

236 occurred, and we witnessed the natural history of a colonizing Kp ST512 isolate.

237 Overall, we report here a wide genetic diversity of $K p$ ST512 in a single patient

238 that underwent low antimicrobial selective pressure. This diversity must be taken in 239 account when trying to infer transmission routes using WGS during nosocomial 240 outbreaks.

\section{Funding}

243 This work was partially funded by the University Paris-Sud, France. LD, TN and RAB 244 are members of the Laboratory of Excellence in Research on Medication and 245 Innovative Therapeutics (LERMIT) supported by a grant from the French National 246 Research Agency (ANR-10-LABX-33), by the Joint Programming Initiative on 247 Antimicrobial Resistance (JPIAMR) DesInMBL [ANR-14-JAMR-002] and by the 248 European Union's Horizon 2020 Research and Innovation Program under Grant 249 Agreement No. 773830 (Project MedVetKlebs, One Health EJP).

\section{Transparency declarations}

253 None to declare.

\section{Acknowledgments}

256 We want to thank Dr A. Tran-Barrail for providing useful information about this clinical 257 case. 
1. Nordmann P, Cuzon G, Naas T. The real threat of Klebsiella pneumoniae carbapenemase-producing bacteria. Lancet Infect Dis 2009; 9: 228-36.

2. Chen L, Mathema B, Chavda KD et al.. Carbapenemase-producing Klebsiella pneumoniae: molecular and genetic decoding. Trends Microbiol 2014; 22: 686-96.

3. Mathers AJ, Peirano G, Pitout JDD. The Role of Epidemic Resistance Plasmids and International High-Risk Clones in the Spread of Multidrug-Resistant Enterobacteriaceae. Clin Microbiol Rev 2015; 28: 565-91.

4. Conte V, Monaco M, Giani T, et al. Molecular epidemiology of KPC-producing Klebsiella pneumoniae from invasive infections in Italy: increasing diversity with 272 predominance of the ST512 clade II sublineage. J Antimicrob Chemother 2016: 273 dkw337.

274 5. Fortini D, Villa L, Feudi C, et al. Double Copies of bla KPC-3::Tn 4401a on an IncX3 275 Plasmid in Klebsiella pneumoniae Successful Clone ST512 from Italy. Antimicrob 276 Agents Chemother 2016; 60: 646-9.

277 6. Cuzon G, Naas T, Truong H, et al. Worldwide Diversity of Klebsiella pneumoniae 278 That Produce $\beta$-Lactamase bla KPC-2 Gene1. Emerg Infect Dis 2010; 16: 1349-56. 7. Naas T, Cuzon G, Villegas M-V, et al. Genetic Structures at the Origin of Acquisition 280 of the $\beta$-Lactamase blaKPC Gene. Antimicrob Agents Chemother 2008; 52: 1257-63.

281 8. Eilertson B, Chen L, Li A,,et al.. CG258 Klebsiella pneumoniae isolates without $\beta$ 282 lactam resistance at the onset of the carbapenem-resistant Enterobacteriaceae epidemic in New York City. J Antimicrob Chemother 2019; 74: 17-21.

9. Conlan S, Park M, Deming C, et al. Plasmid Dynamics in KPC-Positive Klebsiella 285 pneumoniae during Long-Term Patient Colonization. mBio 2016; 7: e00742-16.

286 10. Jousset AB, Bonnin RA, Rosinski-Chupin I, et al. A 4.5-Year Within-Patient 
287 Evolution of a Colistin-Resistant Klebsiella pneumoniae Carbapenemase-Producing K. pneumoniae Sequence Type 258. Clin Infect Dis Off Publ Infect Dis Soc Am 2018; 67: 1388-94.

11. European Committee on Antimicrobial Susceptibility Testing. EUCAST expert rules. 2016. Available at: http://www.eucast.org/.

12. Patiño-Navarrete R, Rosinski-Chupin I, Cabanel N, et al. Stepwise evolution and convergent recombination underlie the global dissemination of carbapenemaseproducing Escherichia coli. Genome Med 2020; 12: 10.

13. Zankari $\mathrm{E}$, Hasman $\mathrm{H}$, Cosentino $\mathrm{S}$, et al. Identification of acquired antimicrobial resistance genes. J Antimicrob Chemother 2012; 67: 2640-4.

14. Seemann T. Prokka: rapid prokaryotic genome annotation. Bioinforma Oxf Engl 2014; 30: 2068-9.

15. Kieser T. Factors affecting the isolation of CCC DNA from Streptomyces lividans and Escherichia coli. Plasmid 1984; 12: 19-36.

16. García-Fernández A, Villa L, Carta C, et al. Klebsiella pneumoniae ST258 producing KPC-3 identified in italy carries novel plasmids and OmpK36/OmpK35 porin variants. Antimicrob Agents Chemother 2012; 56: 2143-5. Types, and Plasmid Analyses of KPC-Producing Klebsiella pneumoniae Strains in Israel. Antimicrob Agents Chemother 2010; 54: 3002-6.

18. Doumith $\mathrm{M}$, Findlay J, Hirani $\mathrm{H}$, et al. Major role of $\mathrm{pKpQIL-like} \mathrm{plasmids} \mathrm{in} \mathrm{the}$ early dissemination of KPC-type carbapenemases in the UK. J Antimicrob Chemother 2017. Available at: https://academic.oup.com/jac/article-

310 lookup/doi/10.1093/jac/dkx141.

311 19. Villa L, Capone A, Fortini D, et al. Reversion to susceptibility of a carbapenem- 
312 resistant clinical isolate of Klebsiella pneumoniae producing KPC-3. J Antimicrob 313 Chemother 2013; 68: 2482-6.

314 20. Peirano G, Bradford PA, Kazmierczak KM, et al. Importance of Clonal Complex 315258 and IncF k2-like Plasmids among a Global Collection of Klebsiella pneumoniae with 316 bla кPC. Antimicrob Agents Chemother 2017; 61: e02610-16. 
Table 1: Clinical features, antimicrobial susceptibility, resistomes and accession numbers of $K$. pneumoniae isolates

\begin{tabular}{|c|c|c|c|c|c|c|c|c|c|c|c|c|c|c|c|c|}
\hline \multirow{2}{*}{ Isolates } & \multirow{2}{*}{ Source } & \multicolumn{9}{|c|}{ Susceptibility testing $(\mathrm{mg} / \mathrm{L})$} & \multicolumn{4}{|c|}{ Resistome } & \multirow[t]{2}{*}{ BioProject } & $\begin{array}{c}\text { Genbank Accession } \\
\text { numbers }\end{array}$ \\
\hline & & CAZ & IPM & ERT & MER & TGC & $\mathrm{COL}$ & GN & AK & $\mathrm{CIP}$ & Chromosome & pKPC & pKPN3-like & pColE & & \\
\hline D1 & rectal & 128 & 4 & $>32$ & $>32$ & 4 & 1 & 1 & 32 & $>32$ & $\begin{array}{c}\text { blasHV-11, gyrA S83I fosA -like, } \\
\text { OmpK35 (truncated), OmpK36 } \\
\text { (mutated) }\end{array}$ & $\begin{array}{c}b / a_{\mathrm{KPC}-3}, b / a_{\mathrm{TEM}-1} \\
\text { bla } \\
\text { OXA-9*, }\end{array}$ & $\begin{array}{l}\text { aph }\left(3^{\prime}\right)-\text {-la, aadA2, sul1, } \\
\text { catA1-like, dfrA12, }\end{array}$ & $a a c\left(6^{\prime}\right)-I b$ & PRJNA564512 & $\begin{array}{|lr|}\text { Chromosome } & \text { CP043969 } \\
\text { pKPN3-like } & \text { CP043970 } \\
\text { pD1-KPC } & \text { CP043971 } \\
\text { pColE } & \text { CP043972 }\end{array}$ \\
\hline D19-1 & rectal & $>256$ & 8 & $>32$ & $>32$ & 4 & 0,5 & 1 & 32 & $>32$ & $\begin{array}{c}\text { b/asHV-11, gyrA S83I fosA -like, } \\
\text { OmpK35 (truncated), OmpK36 } \\
\text { (mutated) }\end{array}$ & $\begin{array}{c}b / a_{\mathrm{KPC}-3}, b / a_{\mathrm{TEM}-1} \\
\text { bla } \\
\text { OXA-9* }\end{array}$ & $\begin{array}{l}\text { aph(3')-la, aadA2, sul1, } \\
\text { catA1-like, dfrA12, }\end{array}$ & $a a c\left(6^{\prime}\right)-I b$ & PRJNA564512 & VWOU00000000 \\
\hline D19-2 & urine & 0,38 & 0.125 & 0,094 & 0,032 & 1 & 1 & 1 & 48 & $>32$ & $\begin{array}{c}\text { bla }_{\mathrm{SHV}-11,} \text { gyrA S83I fosA -like, } \\
\text { OmpK35 (truncated), OmpK36 } \\
\text { (mutated) }\end{array}$ & missing & $\begin{array}{l}\text { aph(3')-la, aadA2, sul1, } \\
\text { catA1-like, dfrA12, }\end{array}$ & $a a c\left(6^{\prime}\right)-1 b$ & PRJNA564512 & VWOV00000000 \\
\hline D26-1 & rectal & $>256$ & 4 & $>32$ & $>32$ & 1,5 & 1 & 1 & 32 & $>32$ & $\begin{array}{c}\text { bla } \text { sHV-11, }_{\text {grrA S83I fosA -like, }} \\
\text { OmpK35 (truncated), OmpK36 } \\
\text { (mutated) }\end{array}$ & $\begin{array}{c}b / a_{\mathrm{KPC}-3}, b / a_{\mathrm{TEM}-1} \\
\text { bla } \\
\text { OXA-9* }\end{array}$ & $\begin{array}{l}\text { aph }\left(3^{\prime}\right) \text {-la, aadA2, sul1, } \\
\text { catA1-like, dfrA12, }\end{array}$ & $a a c\left(6^{\prime}\right)-1 b$ & PRJNA564512 & VWOS00000000 \\
\hline D26-2 & urine & 0,38 & 0.125 & 0,094 & 0,032 & 1 & 1 & 1,5 & 48 & $>32$ & $\begin{array}{c}\text { blastv-11, gyrA S83I fosA -like, } \\
\text { OmpK35 (truncated), OmpK36 } \\
\text { (mutated) }\end{array}$ & missing & $\begin{array}{l}\text { aph }\left(3^{\prime}\right) \text {-la, aadA2, sul1, } \\
\text { catA1-like, dfrA12, }\end{array}$ & $a a c\left(6^{\prime}\right)-1 b$ & PRJNA564512 & VWOT00000000 \\
\hline D33-1 & urine & $>256$ & 6 & $>32$ & $>32$ & 1,5 & 1 & 1 & 48 & $>32$ & $\begin{array}{c}\text { bla } a_{\mathrm{SHV}-11,} \text { gyrA S831 fosA -like, } \\
\text { OmpK35 (truncated), OmpK36 } \\
\text { (mutated) }\end{array}$ & $\begin{array}{c}\text { bla } \boldsymbol{a}_{\mathrm{KPC}-3}, b / a_{\mathrm{TEM}-1} \\
\text { bla } \\
\text { OXA-9* }\end{array}$ & $\begin{array}{l}\text { aph }\left(3^{\prime}\right) \text {-la, aadA2, sul1, } \\
\text { catA1-like, dfrA12, }\end{array}$ & $a a c\left(6^{\prime}\right)-l b$ & PRJNA564512 & VWOP00000000 \\
\hline D33-2 & urine & 0,5 & 0.125 & 0,094 & 0,032 & 1 & 1 & 1,5 & 32 & $>32$ & $\begin{array}{c}\text { b/a } a_{\mathrm{SHV}-11,}, \text { gyrA S83I fosA -like, } \\
\text { OmpK35 (truncated), OmpK36 } \\
\text { (mutated) }\end{array}$ & missing & $\begin{array}{l}\text { aph }\left(3^{\prime}\right) \text {-la, aadA2, sul1, } \\
\text { catA1-like, dfrA12, }\end{array}$ & $a a c\left(6^{\prime}\right)-l b$ & PRJNA564512 & VWOR00000000 \\
\hline D33-3 & rectal & $>256$ & 4 & $>32$ & $>32$ & 1,5 & 1 & 1 & 32 & $>32$ & $\begin{array}{c}\text { blasHV-11, gyrA S83I fosA -like, } \\
\text { OmpK35 (truncated), OmpK36 } \\
\text { (mutated) }\end{array}$ & $\begin{array}{c}b / a_{\mathrm{KPC}-3}, b / a_{\mathrm{TEM}-1} \\
\quad b / a_{\mathrm{OXA}-9^{*}}\end{array}$ & $\begin{array}{l}\text { aph(3')-la, aadA2, sul1, } \\
\text { catA1-like, dfrA12, }\end{array}$ & $a a c\left(6^{\prime}\right)-1 b$ & PRJNA564512 & VWOQ00000000 \\
\hline D40 & rectal & $>256$ & 4 & $>32$ & $>32$ & 1,5 & 1 & 1 & 48 & $>32$ & $\begin{array}{c}b^{\prime} a_{\text {SHV-11, }} \text { gyrA S83I fosA -like, } \\
\text { OmpK35 (truncated), OmpK36 } \\
\text { (mutated) }\end{array}$ & $\begin{array}{c}b / a_{\mathrm{KPC}-3}, b / a_{\mathrm{TEM}-1} \\
\quad \text { bla } \\
\text { OXA-9*, }\end{array}$ & $\begin{array}{l}\text { aph(3')-la, aadA2, sul1, } \\
\text { catA1-like, dfrA12, }\end{array}$ & $a a c\left(6^{\prime}\right)-I b$ & PRJNA564512 & VWOO00000000 \\
\hline D41 & urine & 0,5 & 0.125 & 0,094 & 0,032 & 1 & 1 & 1,5 & 32 & $>32$ & $\begin{array}{c}\text { bla } a_{\mathrm{SHV}-11}, \text { gyrA S83I fosA -like, } \\
\text { OmpK35 (truncated), OmpK36 } \\
\text { (mutated) }\end{array}$ & missing & $\begin{array}{l}\text { aph(3')-la, aadA2, sul1, } \\
\text { catA1-like, dfrA12, }\end{array}$ & $a a c\left(6^{\prime}\right)-l b$ & PRJNA564512 & VWONO0000000 \\
\hline D43 & rectal & $>256$ & 6 & $>32$ & $>32$ & 1 & 1 & 1 & 32 & $>32$ & $\begin{array}{c}\text { bla } a_{\mathrm{SHV}-11}, \text { gyrA S83I fosA -like, } \\
\text { OmpK35 (truncated), OmpK36 } \\
\text { (mutated) }\end{array}$ & $\begin{array}{c}b / a_{\mathrm{KPC}-3}, b / a_{\mathrm{TEM}-1} \\
\quad b / a_{\mathrm{OXA}-9^{*}}\end{array}$ & $\begin{array}{l}\text { aph(3')-la, aadA2, sul1, } \\
\text { catA1-like, dfrA12, }\end{array}$ & $a a c\left(6^{\prime}\right)-1 b$ & PRJNA564512 & VWOM00000000 \\
\hline D54 & rectal & $>256$ & 6 & $>32$ & $>32$ & 1,5 & 0,5 & 1 & 48 & $>32$ & $\begin{array}{c}\text { blas } \text { s }_{\mathrm{HV}-11,} \text { gyrA S83I fosA -like, } \\
\text { OmpK35 (truncated), OmpK36 } \\
\text { (mutated) }\end{array}$ & $\begin{array}{c}b / a_{\mathrm{KPC}-3}, b / a_{\mathrm{TEM}-1} \\
\text { bla } \\
\text { OXA-9* }\end{array}$ & $\begin{array}{c}\text { aph }\left(3^{\prime}\right) \text {-la, aadA2, sul1, } \\
\text { catA1-like, dfrA12, }\end{array}$ & $a a c\left(6^{\prime}\right)-l b$ & PRJNA564512 & VWOL00000000 \\
\hline
\end{tabular}

CAZ: ceftazidime; IPM: imipenem; ERT: ertapenem; MER: meropenem; TGC: tigecyline; GN: gentamicin ; AK: amikacin ; CIP: ciprofloxacin 
A.

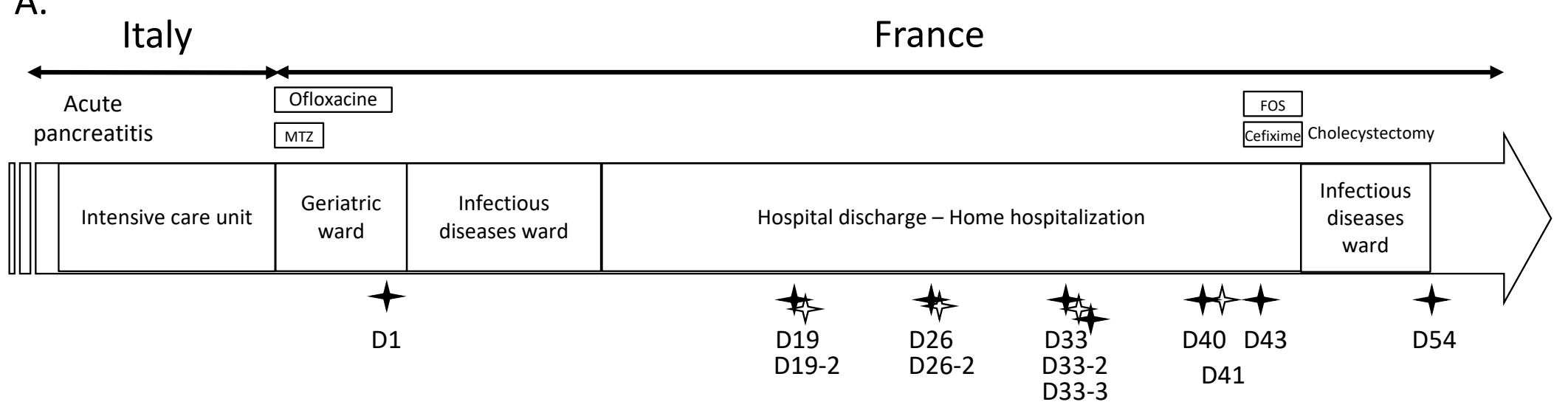

B.

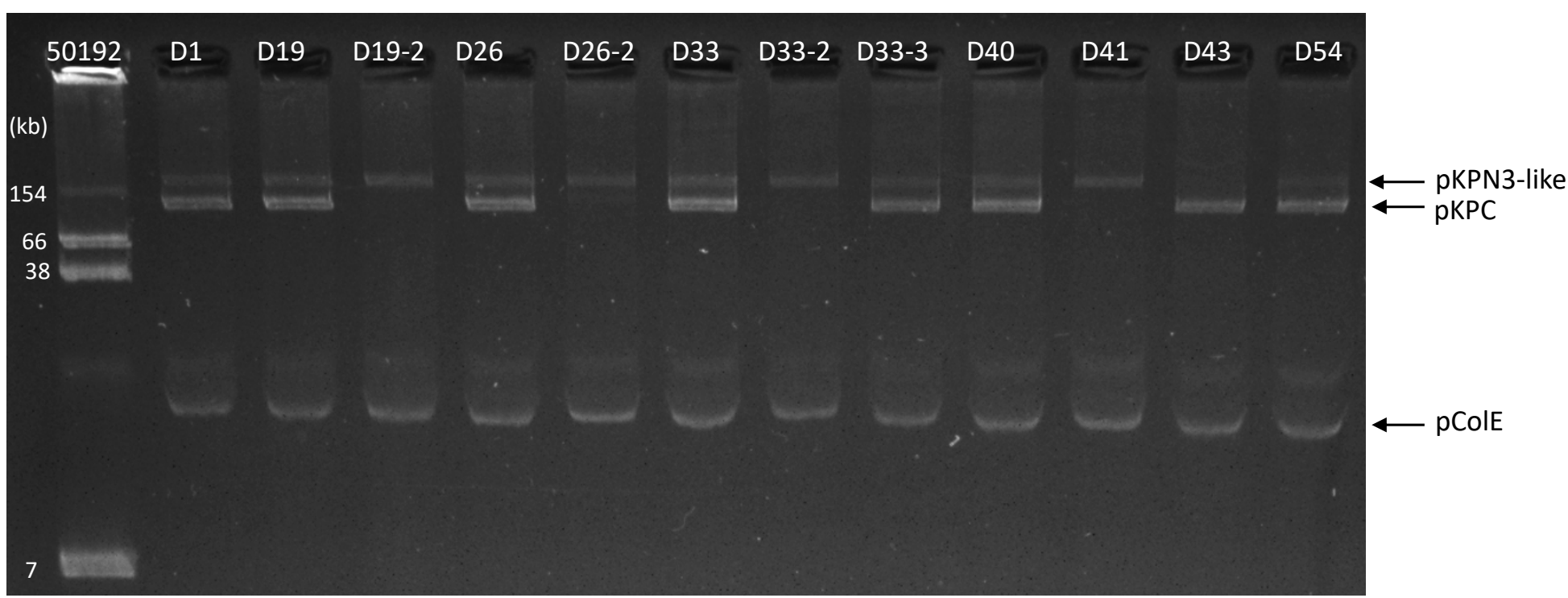


C.

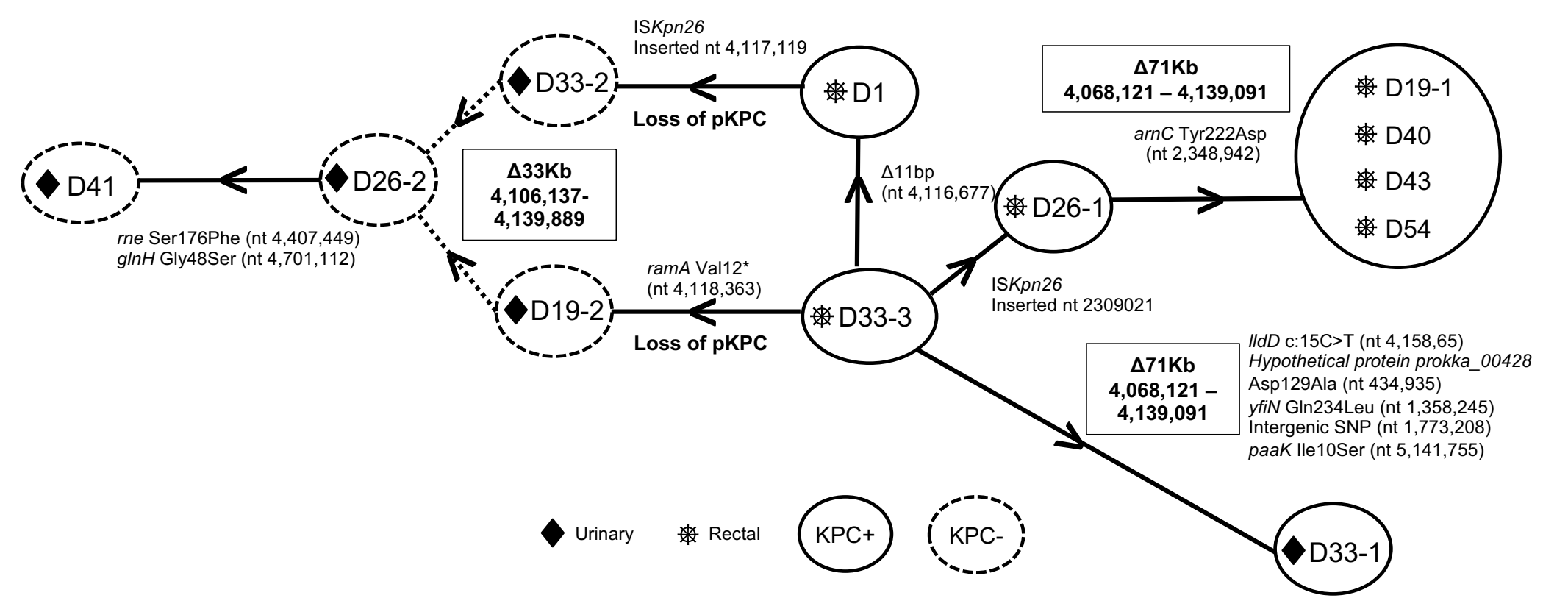

D.

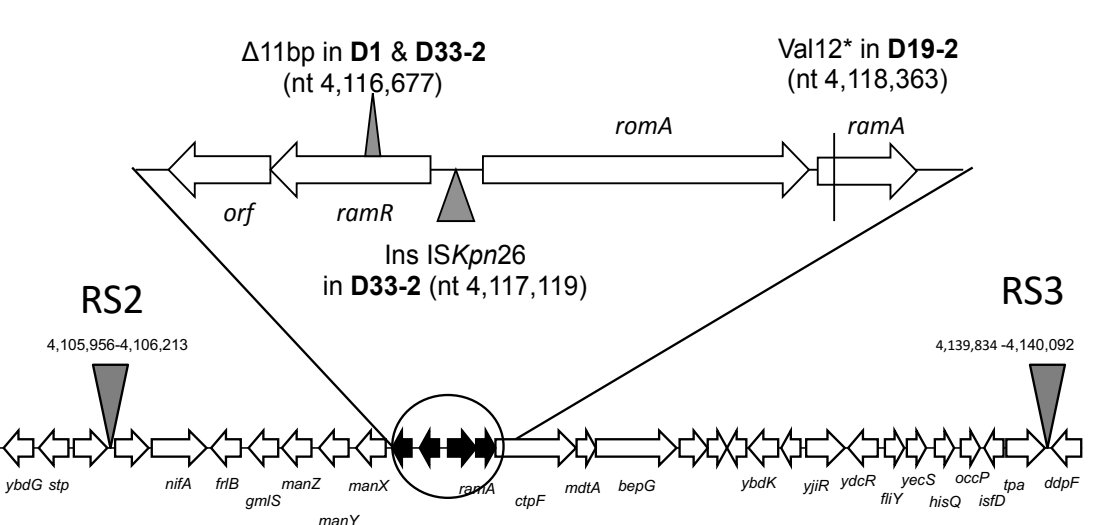

RS1

4,067,866-4,068, 124

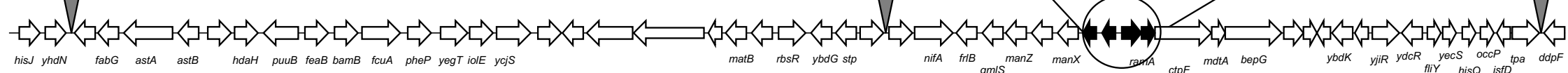

$\Delta 71 \mathrm{~kb}$ in D19-1, D26-1, D33-1, D40, D43, D54 
Figure's legend:

Figure 1. A. Patient's clinical case. This timeline indicates the patient's medical history during the two-months of follow-up. Antibiotics prescribed have been written within boxes. KPC+ and KPC - Kp are indicated by black and white stars respectively. At hospital admission in France, six days of ofloxacin per os combined with intravenous metronidazole for two days were given. After the identification of the first KPC-Kp D1 from rectal swab and since no pancreatitis necrosis could be evidenced, this antimicrobial chemotherapy was stopped. The patient received no antimicrobial treatment until its hospital discharge, when an episode of asymptomatic bacteriuria was treated using Fosfomycin and Cefixime per os between day 44 and day 47 of the follow-up. The timeline was drawn at scale.

B. Plasmid extraction analysed by electrophoresis on a $0.7 \%$ agarose gel. E. coli 50192 was used as reference for plasmid size. The arrows indicate the position of the three plasmids: pKPN3-like, pKPC (missing in KPC- isolates) and pColE.

C. Phylogenetic analysis of isolates recovered over the two-months period. The circles are at scale with the number of isolates. KPC+ and KPC- Kp are indicated in full or in dashed circles respectively. The length of the branches is proportional to the number of genetic events (insertions, deletions, loss of pKPC plasmid, SNPs). Dashed lines indicate two different putative evolutionary pathways.

D. Genetic environment of the deleted regions. Repeated sequences (RS) are indicated by grey triangles. Losses of the $71 \mathrm{~kb}$ and $33 \mathrm{~kb}$ fragments likely occurred after homologous recombination implicating RS1/RS3 and RS2/RS3 respectively. A total of five independent genetic events occurred in this region that contains the ramRromA-ramA genes. 